\title{
Flame Hair: Auxiliary Trichoscopic Finding in Radiotherapy-Induced Alopecia
}

\author{
Ana Beatriz Silva Lima Fernanda Tirelli Rocha Fernanda André Martins Cruz Perecin \\ João Carlos Lopes Simão Andrezza Telles Westin Marco Andrey Cipriani Frade \\ Division of Dermatology, Department of Internal Medicine, Hospital of the clinics of Ribeirão Preto, University of \\ São Paulo, Ribeirão Preto, Brazil
}

\section{Established Facts}

- Radiotherapy is a classic cause of anagen effluvium.

- Flame hairs (FHs) are classically described in trichotillomania but are also observed in other types of alopecia.

- FHs correspond to pigmented hair residues with a fine and short wavy end, resembling a flame.

\section{Novel Insights}

- The presence of flame hairs (FHs) is a valuable finding in the diagnosis of anagen effluvium (AE) secondary to radiotherapy.

- $\mathrm{FH}$ is the most characteristic conformation observed in the trichoscopy of AE.

\section{Keywords}

Anagen · Effluvium · Alopecia · Radiotherapy · Trichoscopy

Abstract

Radiotherapy-induced alopecia is characterized by an anagen effluvium (AE). It may be temporary or permanent, depending on the radiation dose. The diagnosis is based on clinical history and dermatologic exam with trichoscopy being auxiliary the presence of flame hair. We describe a case of an $A E$ induced by radiotherapy, emphasizing the trichoscopic findings. (c) 2021 S. Karger AG, Basel

\section{Introduction}

Radiotherapy-induced alopecia (RIA) is characterized by an acute anagen effluvium (AE) caused by intense damage to the hair follicle. Alopecia starts at 1-3 weeks after the first radiation procedure, and the hair regrowth initiates 2-6 months after the last radiotherapy session. Radiation dermatitis and severe skin damage may also occur.

RIA is due to dose-dependent damage to the matrix cells of anagen follicles [1-3]. The trichoscopic findings are very similar to those of alopecia areata [3], include black dots, yellow dots, short vellus hair, peripilar signal, flame hairs (FHs), and other types of broken hairs. FHs can be suggestive of RIA and represent pigmented hair residues resembling a flame [4].
Ana Beatriz Silva Lim

Department of Internal Medicine, Division of Dermatology Hospital of Clinics of Ribeirão Preto, University of São Paulo Ave. Bandeirantes, 3900, Ribeirão Preto 14015-010 (Brazil) anabslima@gmail.com 


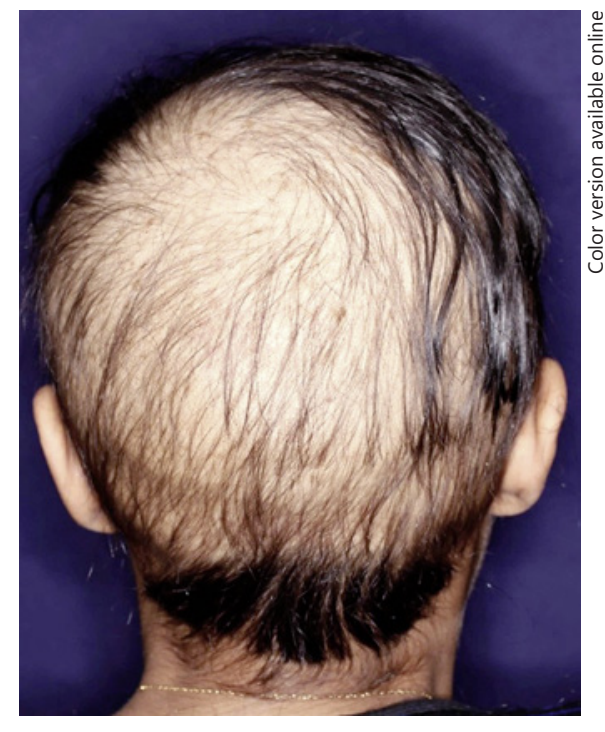

Fig. 1. Extensive alopecia in the occipital region.

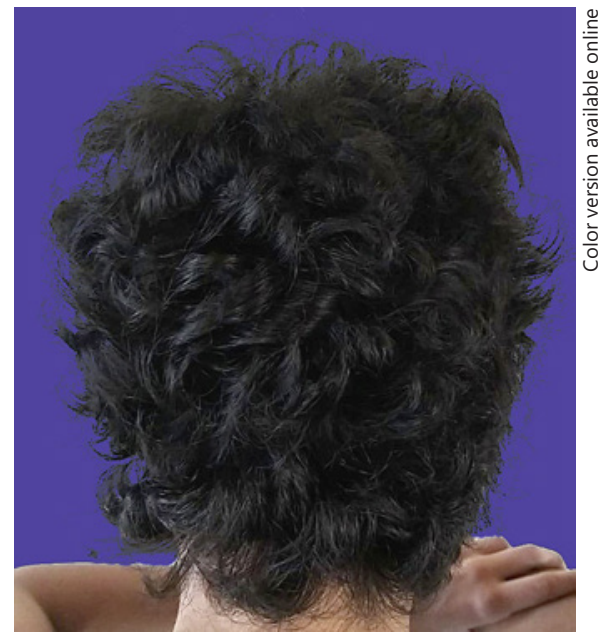

Fig. 3. Complete repilation of the occipital regions.

\section{Case Presentation}

A 23-year-old female patient diagnosed with papillary lung adenocarcinoma with bone and central nervous system metastasis for 2 months was referred to the dermatology service due to the diffuse and abrupt alopecia. The patient was submitted to radiotherapy of the central nervous system with a total dose of $20 \mathrm{~Gy}$. One week after the last session, she developed rapidly progressive hair shedding, and after 7 days she already presented diffuse alopecia. She had no hair loss on her body or eyebrows. On physical examination, she presented with diffuse alopecia, more intense in the temporal and occipital regions (shown in Fig. 1). The pull test was positive. Yellow and black dots, broken hairs, and FHs were observed in large quantities in trichoscopy (shown in Fig. 2). The

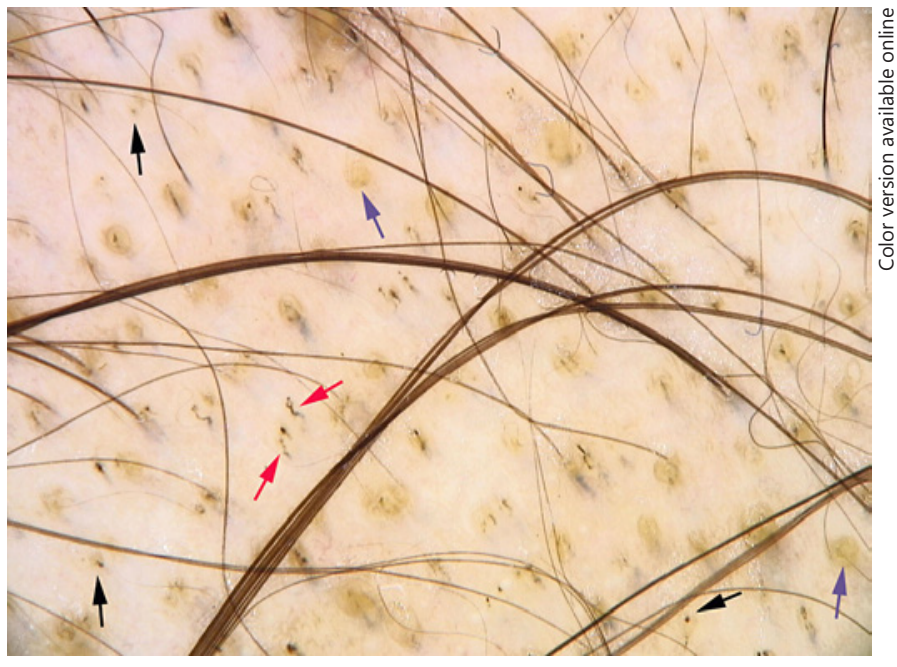

Fig. 2. Trichoscopy showing black (black arrows) and yellow (blue arrows) dots. Presence of FHs (red arrows) (FotoFinder Systems, $\times 20$ magnification). FHs, flame hairs.

diagnostic hypothesis was AE due to radiotherapy. Alcoholic solution of $5 \%$ minoxidil was prescribed once a day. After 3 months, the patient presented with repilation of some areas of the scalp, negative pull test and, short regrowing hairs were observed in trichoscopy. Eight months after the first appointment, the patient evolved with total hair regrowth, demonstrating follicular recovery without permanent damage (shown in Fig. 3).

\section{Discussion/Conclusion}

In the $\mathrm{AE}$, hair loss is the result of follicular aggression suffered in the anagen phase leading to an injury in the mitotic activity of the matrix cells. Follicular bulb cells have high mitotic activity in the anagen phase and are more sensitive to cytotoxic aggression. There is a narrowing of the proximal portion of the hair shaft, which becomes fragile and susceptible to fracture. The most common causes of $\mathrm{AE}$ are chemotherapy and radiotherapy [3].

Patients with AE usually have non-scarring alopecia that begins 7-14 days after exposure to the injury factor, extending over 3-4 weeks [1]. Radiotherapy-induced AE can lead to temporary or permanent alopecia with irreversible follicular damage. A study conducted in patients with brain metastases showed a $50 \%$ risk of permanent alopecia with a fractional dose equal or greater than $43 \mathrm{~Gy}$ [5]. In the case of a single radiation dose, values $>7$ Gy were also associated with definitive damage. In addition to the dose, the type and depth of the radiation site are decisive in the time and evolution of alopecia [3]. Our 
patient received 20 Gy in a fractionated way, thus lowering the probability of definitive alopecia.

The clinical presentation of alopecia ranges from geometric shapes confined to the irradiated areas to diffuse shapes when the entire head is exposed. Dermatitis by radiotherapy and intense skin damage may also be associated. The histopathologic examination shows a reduction in the follicular bulb volume and an increase in the catagen/telogen ratio, without inflammation. The diagnosis is made by clinical history and physical examination with trichoscopy $[1,3]$.

The predominant trichoscopic findings are yellow and black dots (60\%), vellus hair (50\%), peripilar signal (20\%) and broken hairs (10\%). The most characteristic type of broken hair is $\mathrm{FH}$ [1]. Although the presence of AE in an adequate clinical context is sufficient for the diagnosis of RIA, the trichoscopic findings can assist in the etiological definition.

FH were initially described by Rakowska et al. [6] as a trichoscopic sign of trichotillomania, resulting from severe external damage to the hair shaft, secondary to the act of mechanically pulling the anagen hairs. FHs correspond to pigmented pilar residues with thin and wavy end, resembling a flame. In the anatomopathological examination, they appear as individual hair follicles with distorted hair shafts at the level of the infundibulum. Miteva and Tosti, in 2015, described that this trichoscopic alteration is not exclusive to trichotillomania and can be found in several other pathologies such areata alopecia, traction alopecia, and central centrifugal cicatricial alopecia. In their study, FH were found in $100 \%$ of acute chemotherapy- and RIA, representing the predominant type of broken hairs [4]. Our patient presented trichoscopy with multiple $\mathrm{FH}$, as well as numerous yellow and black dots (shown in Fig. 1, 2 ), which associated with the clinical history corroborate the diagnosis of $\mathrm{AE}$ associated with radiotherapy.

The literature presents as therapeutic options the daily use of 5\% minoxidil and topical corticosteroid, in case of association with radiation dermatitis[7, 8]. We present a case of $\mathrm{AE}$ induced by radiotherapy emphasizing the trichoscopic findings and the most relevant clinical aspects in this type of alopecia.

\section{Acknowledgements}

The authors thank the members of the division of Dermatology of Hospitsal of Clinics of Ribeirao Preto.

\section{Statement of Ethics}

This case report was approved by the Research Ethics Committee under the protocol number 4.188 .722 . The patient provided a free and informed consent form for publication of the case, including her images.

\section{Conflict of Interest Statement}

The authors have no conflicts of interest to declare.

\section{Funding Sources}

No funding sources to this report.

\section{Author Contributions}

Ana Beatriz Silva Lima and Fernanda Tirelli Rocha: patient care, article making, bibliographic reviewer. Fernanda André Martins Cruz Perecin and João Carlos Lopes Simão: dermatologists who served as advisors to the study and as article reviewers and helped in correction and improvement of the scientific article. Andrezza Telles Westin: dermatologist who served as an advisor to the study and as an article reviewer and translator and helped in scorrection and improvement of the scientific article. Marco Andrey Cipriani Frade: served as the teaching supervisor and as an article reviewer for the study and responsible for the correction and improvement of the scientific article.

\section{References}

1 Freites-Martinez A, Shapiro J, Goldfarb S, Nangia J, Jimenez JJ, Paus R, et al. Hair disorders in patients with cancer. J Am Acad Dermatol. 2019;80(5):1179-96.

2 Kanwar AJ, Narang T. Anagen effluvium. Indian J Dermatol Venereol Leprol. 2013;79(5):604-12.

3 Suchonwanit P, McMichael AJ. Alopecia in association with malignancy: a review. Am J Clin Dermatol. 2018;19(6):853-65.
4 Miteva M, Tosti A. Flame hair. Skin Appendage Disord. 2015;1(2):105-9.

5 Freites-Martinez A, Shapiro J, van den Hurk C, Goldfarb S, Jimenez JJ, Rossi AM, et al. Hair disorders in cancer survivors. J Am Acad Dermatol. 2019;80(5):1199-213.

6 Rakowska A, Slowinska M, Olszewska M, Rudnicka L, et al. New trichoscopy findings in trichotillomania: flame hairs, V-sign, hook hairs, hair powder, tulip hairs. Acta Derm Venereol. 2014;94:303-6.

7 Ali SY, Singh G. Radiation-induced alopecia. Int J Trichology. 2010;2(2):118-9.

8 Metz JM, Smith D, Mick R, Lustig R, Mitchell J, Cherakuri M, et al. A phase I study of topical Tempol for the prevention of alopecia induced by whole brain radiotherapy. Clin Cancer Res. 2004;10(19):6411-7. 